\title{
Norois
}

Environnement, aménagement, société

197 | 2005/4

Varia

\section{Vers une analyse contextualisée des comportements électoraux. Le cas de l'agglomération caennaise en 2002}

Towards a contextual analysis of the electoral behaviours. The case of the urban area of Caen in 2002

\section{Jean Rivière}

\section{OpenEdition}

Journals

Édition électronique

URL : https://journals.openedition.org/norois/345

DOI : $10.4000 /$ norois. 345

ISBN : 978-2-7535-1545-1

ISSN : 1760-8546

\section{Éditeur}

Presses universitaires de Rennes

\section{Édition imprimée}

Date de publication : 1 décembre 2005

Pagination : 95-106

ISBN : 978-2-7535-0238-3

ISSN : 0029-182X

Référence électronique

Jean Rivière, «Vers une analyse contextualisée des comportements électoraux. Le cas de

l'agglomération caennaise en 2002 », Norois [En ligne], 197 | 2005/4, mis en ligne le 15 décembre 2008, consulté le 13 janvier 2022. URL : http://journals.openedition.org/norois/345 ; DOI : https:// doi.org/10.4000/norois.345

Ce document a été généré automatiquement le 13 janvier 2022.

(c) Tous droits réservés 


\section{Vers une analyse contextualisée des comportements électoraux. Le cas de l'agglomération caennaise en 2002}

Towards a contextual analysis of the electoral behaviours. The case of the urban area of Caen in 2002

Jean Rivière

\section{NOTE DE L'ÉDITEUR}

Cet article a été reçu le 9 juin 2005 et définitivement accepté le $1^{\text {er }}$ décembre 2005.

\footnotetext{
« Dans l'espace concret, la politique se fabrique très différemment de la société et de l'économie.

Les règles d'apparition d'une opinion et d'un parti [...] possèdent une logique propre qui ne répond pas à celle des règles de création, développement et fermeture des entreprises ni aux différenciations des classes sociales dans

l'espace. " H. Le Bras (2002)
}

Dans Une autre France - premier ouvrage proposant des éléments d'explication des résultats du dernier scrutin présidentiel - le démographe H. Le Bras (2002) relance le débat permanent sur les traductions politiques, et plus largement sociales, des appartenances de classe... Du côté des politologues, l'ouvrage Le nouveau désordre électoral (Cautrès et Mayer, 2004) insiste sur l'apparente volatilité du comportement électoral des électeurs français. Dans un article du Monde au sein duquel ils dressent une analyse des élections régionales de 2004, les économistes D. Goux et E. Maurin 
(2004) écrivent pourtant: «La composition sociale d'une commune ou d'un territoire représente aujourd'hui encore un facteur explicatif majeur de la structure du vote qui s'y exprime. » Ces trois lectures post-électorales récentes, menées par des chercheurs de disciplines différentes, donnent ainsi à lire la pluralité des points de vue sur cette question.

2 Chez les géographes, les choses bougent également. Alors que la géographie électorale française connaît un regain d'intérêt depuis une vingtaine d'années et que «la ségrégation urbaine permet de voir émerger les relations entre la place dans l'échelle sociale et les opinions politiques" (Bon et Cheylan, 1988), peu d'études avaient été menées en géographie électorale urbaine jusqu'à récemment. Plusieurs recherches ont partiellement comblé ces lacunes. Certains travaux mettent l'accent sur les relations entre les politiques d'urbanisme et l'évolution des votes (Badariotti, 1994). D'autres se focalisent sur les liens entre identités politiques locales et stratégies résidentielles en milieu urbain (Lévy, 1994). D'autres encore proposent d'utiliser le vote comme variable d'opinion permettant une approche originale des ségrégations urbaines, ce dans la mesure où les approches de la division sociale de l'espace urbain sont souvent fondées sur des indicateurs sociaux traduisant un état (Girault, 2000).

3 Ce qui suit s'inscrit à la fois dans le champ de la géographie sociale et dans le champ des disciplines qui envisagent les questions politiques de manière plus centrale. Il s'agit essentiellement de proposer une image de la répartition des comportements électoraux dans l'agglomération caennaise, et de mettre en évidence quelques-uns des déterminants sociaux locaux de ces expressions politiques. Dans cette perspective, on avance que les positions socio-économiques des individus et des groupes sociaux dans la structure sociale locale jouent toujours le premier rôle dans l'explication des comportements électoraux, même si ce pouvoir explicatif s'est complexifié, tout comme les contours des catégories sociales.

4 À l'inverse de l'étude du vote telle qu'elle est pratiquée grâce aux sondages d'intention de vote ou post-électoraux (fondés sur des échantillons nationaux redressés pour être représentatifs), on considère que l'interprétation des comportements électoraux ne peut ignorer le contexte historique et géographique au sein duquel l'acte du vote a lieu, "sous peine d'observer des populations trop abstraites, en quelque sorte désincarnées » (Rapetti, 1985). De plus, le fait de travailler non pas sur des données de sondages (pratique déclarée) mais sur le vote lui-même (résultats effectifs) rend possible les analyses exhaustives et cartographiques à des échelles très fines. Cette posture n'implique pas le retour à des collections de monographies ou d'atlas locaux, elle traduit plutôt la volonté de pratiquer une géographie sociale envisageant les votes comme expressions de formations socio-spatiales, qui sont forcément locales. On se propose ainsi d'explorer l'agglomération de Caen lors des dernières élections présidentielles de $2002^{1}$, tout en étant attentif aux consultations électorales passées (élections municipales notamment). 


\section{L'agglomération caennaise à travers le prisme du politique}

\section{De Siegfried à la Normandie actuelle}

Les discours tenus à l'occasion des soirées électorales (et les représentations que ces discours participent à produire) insistent généralement sur le fait que la BasseNormandie constitue un "fief conservateur ", une «terre historiquement favorable à la droite ", etc. Par rapport à cette tendance, la capitale bas-normande présente pourtant des spécificités notables, à tel point qu'A. Frémont (1986) a intitulé "Caen, la charnière ", un chapitre de sa contribution à la Géopolitique des régions françaises.

Dans son célèbre Tableau politique de la France de l'Ouest, A. Siegfried (1913), livre une description minutieuse de Caen : "C'est une ville de basoche, d'étudiants, de rentiers, de moyen et de petit commerce, de cheminots et de déchargeurs de navires. Ce sera prochainement un grand centre industriel, avec ses hauts fourneaux de Colombel et l'exploitation de ses minerais. Mais c'est surtout la métropole historique basnormande, cité des cathédrales romanes et des grands palais administratifs, évoquant les unes comme les autres la même stabilité, de richesse assise et d'ordre traditionnel qu'il en devient harmonieux... C'est enfin - et par là combien si différente de Rouen la réservée - une cité sans mélancolie, capable d'expansion dans l'entraînement parfois bruyant de ses emballements populaires, où derrière la façade bourgeoise se révèle à certains moments je ne sais quelle inquiétante grossièreté de plèbe. »

7 Dès le début $\mathrm{du} \mathrm{xx}^{\mathrm{e}}$ siècle, le père fondateur de la géographie électorale française pressent, à travers l'opposition entre le profil bourgeois de Caen et l'arrivée future de nombreux ouvriers dans les périphéries caennaises, ce qui deviendra une caractéristique sociopolitique fondamentale de l'agglomération. A. Mahé (1968) et A.-M. Seronde-Babonaux (1983) ont ensuite confirmé les intuitions d'A. Siegfried en révélant les contrastes qui caractérisent l'expression électorale des habitants de l'agglomération. Plus récemment, M. Bussi (1992) a souligné, dans sa typologie politique des villes normandes, que Caen se maintient à droite avec une stabilité remarquable pour une ville de son rang, mais qu'il existe un fossé important avec les principales communes satellites marquées, à l'inverse, très à gauche sur le plan politique. Cela dit, les travaux précédemment évoqués étant fondés sur des analyses au niveau communal (au mieux selon les grands quartiers administratifs de la commune de Caen), la problématique mérite d'être traitée à une échelle plus fine : celle des bureaux de vote (fig. 1). 


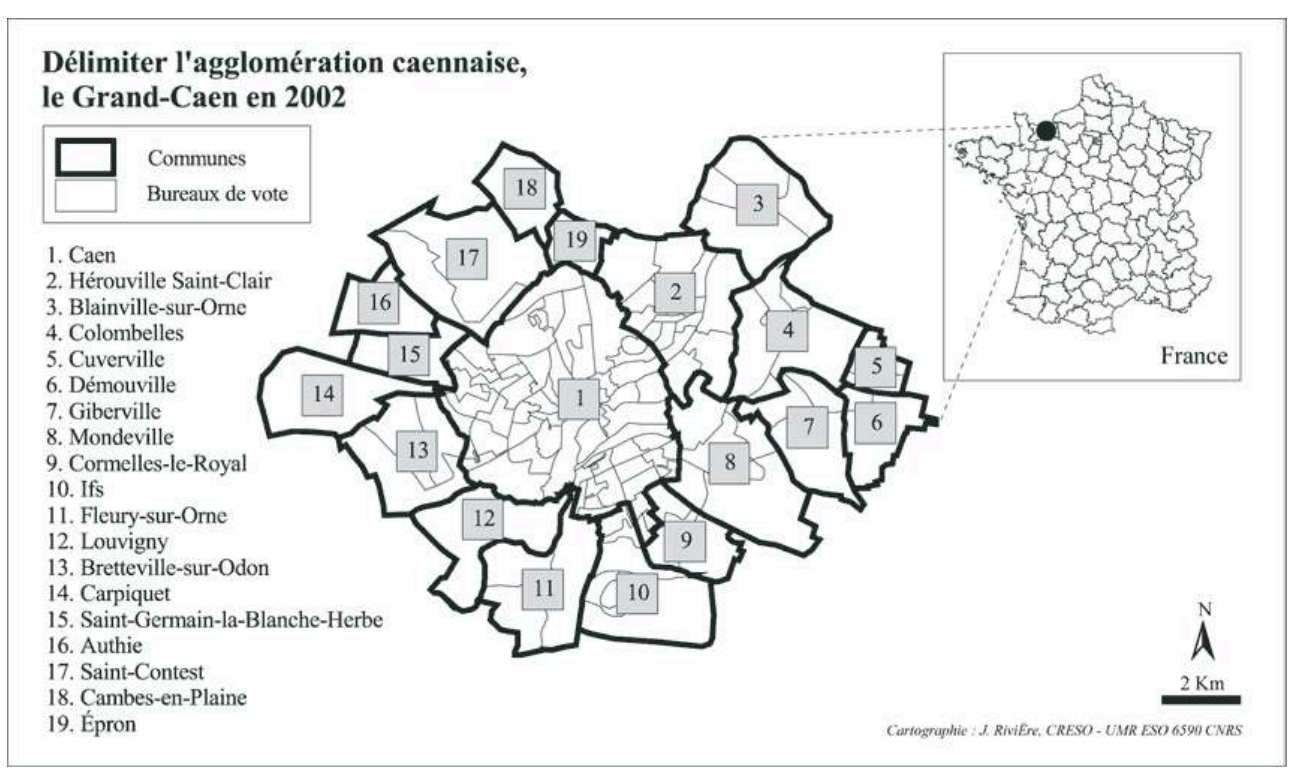

Figure 1 : Localisation de l'espace d'étude

Study area

\section{Quelques éléments méthodologiques}

Dans son manuel La Géographie politique, A.-L. Sanguin (1977) préconise justement le bureau de vote comme échelon d'analyse. Comme le souligne F. Girault (2000), l'inadéquation entre les espaces d'agrégation des données électorales (la délimitation des sections de vote est du ressort des différentes municipalités de l'agglomération) et des données sociales (le recueil des données du recensement de la population est organisé par l'INSEE à l'échelle des îlots puis des IRIS) impose un traitement préalable afin de construire une correspondance entre les deux trames géographiques, ce qui autorise ensuite le calcul de corrélations statistiques.

L'intérêt de ce niveau d'agrégation des données ne renvoie donc pas seulement au fait de pouvoir observer de manière fine la répartition intra-urbaine des comportements électoraux. Le choix du bureau de vote permet également de limiter le risque d'erreur écologique, risque souvent signalé en géographie électorale lorsqu'il s'agit d'interpréter le croisement des résultats des scrutins avec les variables sociodémographiques dont on teste la dimension explicative. Sur ce point, C. Rhein (1994) précise qu'il ne s'agit pas tant de limiter le risque d'erreur écologique (risque qui réside dans le fait d'attribuer à un individu les propriétés constatées au niveau des unités spatiales où les votes sont agrégés) que de veiller à bien hiérarchiser le rôle des différentes données utilisées: "l'erreur écologique est moins un problème d'ordre strictement statistique qu'un problème de méthode pour les spécialistes des sciences sociales : elle ne fait que mettre à nu une erreur de conception de l'objet, erreur que l'utilisateur apprend à éviter par un patient travail sur le rapport entre ses concepts et les variables mises en œuvre. »

\section{Paysages électoraux contemporains}

10 Aux lendemains du premier tour de l'élection présidentielle de 2002, les lecteurs basnormands de Ouest-France pouvaient lire dans les colonnes de leur quotidien: 
«L'agglomération caennaise a été secouée par ses extrêmes²», ce titre associant implicitement les électeurs des périphéries urbaines caennaises et leur expression politique supposée se situer aux périphéries de l'échiquier politique français... Avec un peu de recul, quels sont les grands traits des comportements électoraux des habitants de l'agglomération caennaise par rapport aux tendances nationales relevées en 2002 ?

11 En termes de participation électorale, le profil de l'ensemble urbain est relativement proche du résultat national. Pour ce qui est des différents types de vote, les habitants de l'agglomération ont crédité la gauche de 10 points de plus que la moyenne nationale. $\mathrm{Au}$ sein de la gauche, ce sont surtout les socialistes ( $+5,3$ points) et l'extrême gauche (+3,5 points) qui obtiennent de meilleurs scores. À droite, les résultats sont globalement conformes aux tendances nationales, quoique légèrement défavorables au candidat $\mathrm{J}$. Chirac. En revanche, l'agglomération caennaise est beaucoup moins concernée par le vote d'extrême droite (-6,5 points) que l'ensemble du territoire français. À l'échelle de l'agglomération, des nuances apparaissent entre le comportement électoral des habitants de Caen (plus ancré à droite et moins orienté vers les candidats issus des marges de l'échiquier politique) et celui des résidents des communes périphériques (marqué par de meilleurs scores pour l'extrême gauche et pour l'extrême droite).

12 Afin de dépasser les constats basés sur l'opposition centre-périphérie pour ce qui est de la dimension spatiale, ou sur l'état du rapport gauche-droite en ce qui concerne le champ politique, la réalisation d'une typologie des bureaux de vote (fig. $2 a$ et $2 b$ ) offre un instantané, saisi en 2002, du paysage électoral de l'agglomération. Les trois tendances que cette typologie met en évidence sont assez significatives des principaux clivages politiques observés lors de la dernière consultation présidentielle. On retrouve ainsi, localement, une forme de tripartition de la scène politique nationale, cette tripartition étant fondée sur la montée des comportements dits protestataires (types $1 \mathrm{~A}$ et $1 \mathrm{~B}$ de la $\mathrm{CAH}$ ) d'une part, et sur les deux pôles du clivage gauche (types $2 \mathrm{~A}, 2 \mathrm{~B}$, et $2 \mathrm{C}$ )/droite (types $3 \mathrm{~A}$ et $3 \mathrm{~B}$ ) généralement utilisé en sciences politiques d'autre part. 


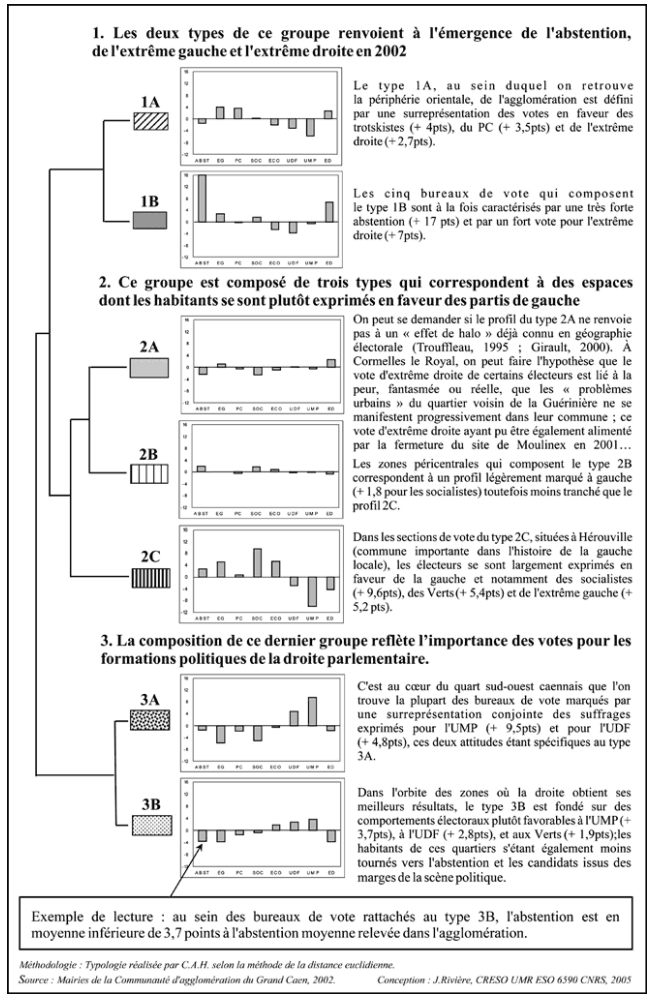

Figure 2a: Typologie électorale des bureaux de vote de l'agglomération caennaise (1er tour de l'élection présidentielle de 2002)

Electoral typology of the polling disctricts in the urban area of Caen (1st turn of the 2002's presidential election)

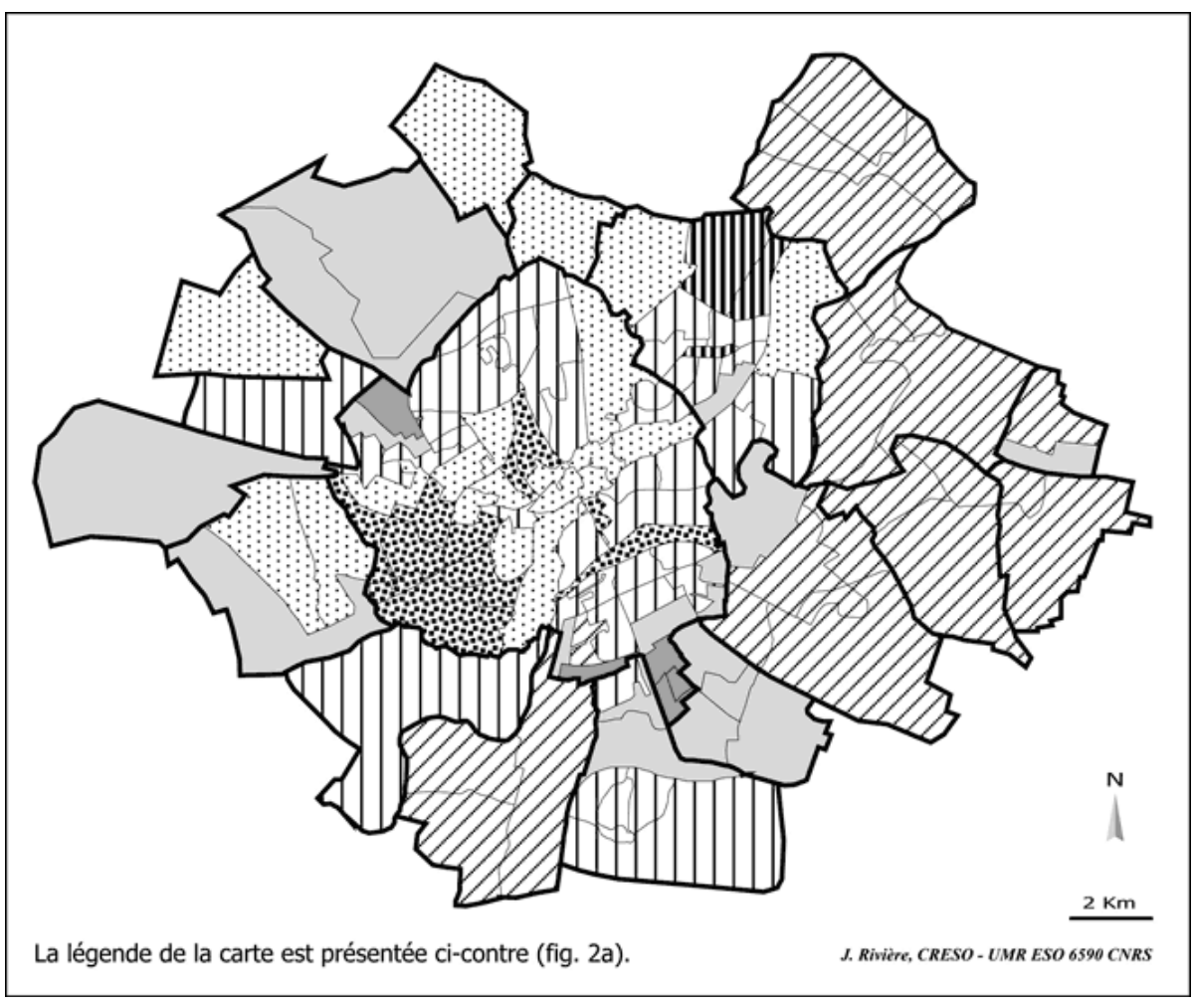

Figure $2 \mathrm{~b}$ : Cartographie de la typologie électorale des bureaux de vote de l'agglomération caennaise (1er tour de l'élection présidentielle de 2002)

Cartography of the electoral typology of the polling disctricts in the urban area of Caen (1st turn of the 2002's presidential election) 
La répartition géographique des comportements électoraux est donc fortement différenciée dans l'espace intra-urbain. Le vote en faveur de certains courants politiques y est tantôt réparti de manière apparemment diffuse, tantôt de façon très concentrée et localisée. Au-delà et dans une perspective de géographie sociale attentive aux divisions sociales de l'espace et aux enjeux de pouvoir localisés, il convient de décrypter les réalités sociales qui sous-tendent ces expressions électorales et politiques.

\section{Derrière la géographie des votes... des dynamiques sociales}

\section{Les comportements électoraux comme marqueurs des divisions sociales de l'espace urbain}

Les analyses qui visent à établir des relations entre les comportements électoraux et le profil social des électeurs sont généralement construites à partir de données nationales. Cependant ces analyses ne donnent pas, pour telle région ou pour tel quartier urbain, les modalités des relations statistiques entre la structure sociale locale et les votes, et surtout elles ne disent pas si l'intensité de ces relations diffère d'une zone à l'autre. Le calcul de corrélations statistiques selon les 118 bureaux de vote de l'agglomération caennaise et la représentation des coefficients de corrélations sous forme de schémas (fig. 3) permet de mettre en lumière les nébuleuses sociales locales à l'origine des différentes attitudes électorales.
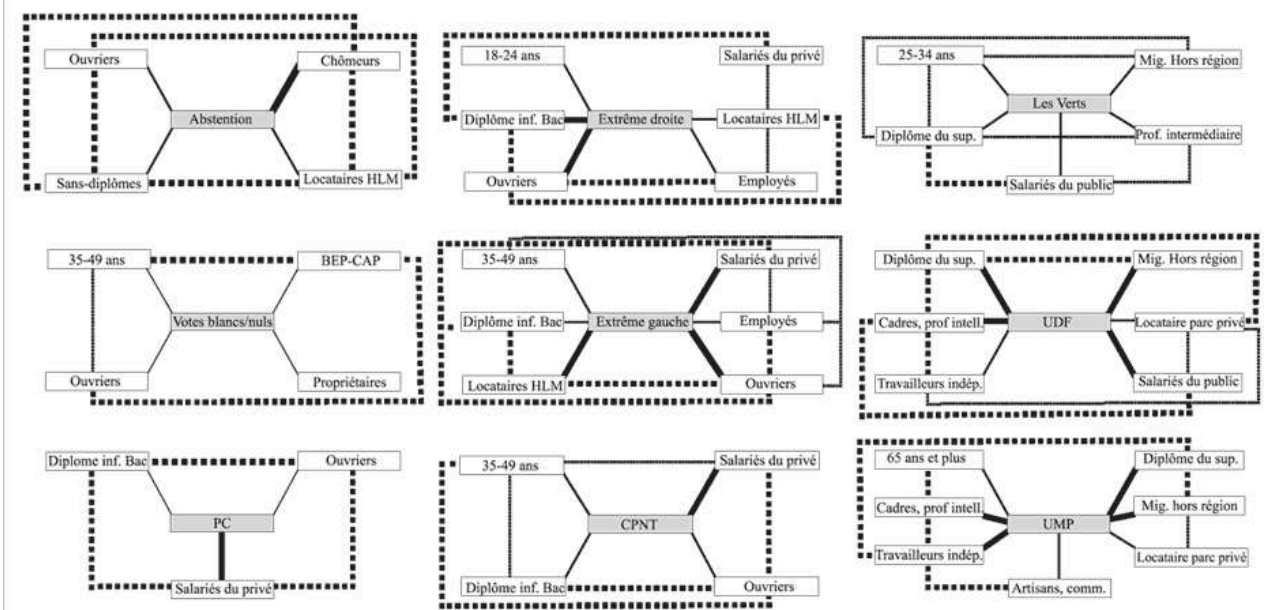

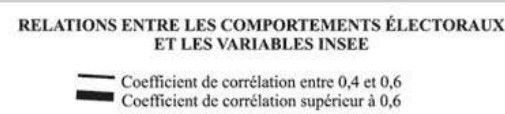

RELATIONS DES VARIABLES INSEE ENTRE-ELLES

Coefficient de corrélation entre 0,4 et 0,6 .... Coefficient de corrálation supérieur à, 0,6
LISTE DES VARIABLES DU RECENSEMENT DE 1999 MOBILISÉES AGE (18-24ans, 25-34ans, 35-49ans, 50-64ans, 65ans et plus)

NIVEAU DE DIPLOME (avcun diptôme, BEP-CAP, diplóme inf. Bac, diplóme du superieur) CSP (artisans commercants, eadres et prof. Intell,., Prof. Intemnédiaires, employés, ouvriers, chômeurs) STATUT SOCIOPROFESSIONNEL (Travailleurs indépendants, salaries du public, salaries du privess) STATUT D'OCCUPATION DU LOGEMENT (proprictaire, locataire parc privé, locataire parc HLM LIEU DE RÉSIDENCE EN 1990 (méme logenent, méme cominune, méme dept., méme région, hoos-région)

Figure 3 : Les bases sociales des différents comportements électoraux (schémas récapitulatifs des corrélations)

Social basis of the electoral behaviours (summary schemes of correlations)

L'inscription sur les listes électorales (que l'on considère souvent comme le premier degré de participation au système électif), l'abstention, le vote blanc ou nul sont 
rarement étudiés comme données sociopolitiques à part entière, sauf peut-être lors des soirées électorales où les commentateurs de la vie politique analysent l'abstention pour tenir en haleine le public dans l'attente de ce que ces journalistes considèrent être les vrais résultats.

Pour ce qui est de la non-inscription, les travaux d'A. Laurent (1997) ont par exemple montré qu'il faut en moyenne trois ans à un nouvel arrivant au sein d'un espace pour s'inscrire sur les listes électorales de sa commune. On peut ainsi faire l'hypothèse que l'intégration au système électoral (ici l'inscription sur les listes électorales) résulte, pour partie, de l'intégration socio-spatiale des populations. Dans l'agglomération caennaise, la non-inscription sur les listes électorales est en effet corrélée négativement $(-0,48)$ avec la présence de populations propriétaires de leur logement. La relation est par contre positive avec la présence des $18-24$ ans $(+0,59)$ qui correspondent à une classe d'âge généralement moins politisée que l'ensemble du corps électoral, et avec les étudiants $(+0,56)$ qui forment une catégorie de population très mobile géographiquement et dont les membres font moins souvent la démarche de s'inscrire sur les listes électorales (peut-être parce qu'ils savent qu'ils vont déménager seulement quelques années après cette inscription, ou préfèrent être inscrits ailleurs) ${ }^{3}$.

L'importance de l'ancrage à l'espace social s'exprime également en ce qui concerne l'abstention puisque que le non-vote est corrélé positivement $(+0,64)$ avec la présence de populations ayant connu une mobilité intra-communale lors de la période intercensitaire, et très négativement avec les propriétaires de leur logement $(-0,71)$. Quant au lien positif $(+0,52)$ entre l'abstention et la présence de population vivant dans le parc HLM, il peut indiquer un désintérêt des milieux populaires vis-à-vis du politique, attitude dont on peut faire l'hypothèse qu'elle exprime un sentiment de relégation. La non-intégration au système politique serait, dans ce cas, un indicateur d'une position de marge, la corrélation avec le pourcentage de chômeurs de moins de un an $(+0,64)$ et de plus de un an $(+0,68)$ est à ce titre intéressante.

18 La géographie du vote blanc ou nul dans l'agglomération caennaise (cette attitude électorale est particulièrement observable au sein de la façade orientale marquée par le fait industriel) et les corrélations qui se dégagent entre cette attitude et les variables sociales ( $r=0,59$ avec les diplômes d'un BEP ou CAP, et 0,51 avec les ouvriers) pousse à s'interroger sur l'influence possible de l'encadrement politique, syndical et associatif existant dans ces communes de tradition ouvrière. On peut en effet penser que cet encadrement participe à faire perdurer la discipline républicaine du vote (qui passe par le bulletin blanc ou nul) parmi des électeurs souhaitant exprimer une protestation à l'égard du système politique.

19 Les votes en faveur des formations situées aux marges de l'échiquier politique (extrême gauche et extrême droite) ont des bases sociales très proches qui renvoient à la présence des populations les moins favorisées socialement (ouvriers et employés dans une moindre mesure, populations disposant d'un diplôme inférieur au niveau Bac, locataires du parc HLM). Le vote pour ces partis en périphérie de la scène politique paraît reposer sur une position sociale plutôt peu favorisée, position se traduisant par le fait de résider dans des espaces périphériques. Les résultats du PC et du CPNT semblent eux aussi liés à la présence de ces mêmes catégories sociales, mais de manière moins nette.

20 À l'inverse, on retrouve les couches sociales aisées (cadres et travailleurs indépendants, diplômés de l'enseignement supérieur, locataires du parc privé) à la fois dans l'électorat 
de l'UDF et de l'UMP ; les artisans et la tranche d'âge des plus de 65 ans étant les seules catégories corrélées au vote UMP de façon significative. Là, le vote pour les formations politiques de la droite gouvernementale semble lié à une forte intégration sociale qui permet à ces populations d'accéder à des localisations résidentielles fondées sur la centralité urbaine. Les scores des Verts sont, quant à eux, liés à la présence des groupes sociaux à partir desquels les politologues expliquent généralement le vote écologiste (salariés du public, diplômés du supérieur, tranche d'âge des 25-34 ans). À noter, par contre, que les résultats du PS ne sont corrélés significativement avec aucune variable INSEE utilisée. La compréhension des votes dans l'agglomération nécessite donc l'analyse d'autres faits sociaux...

\section{Les comportements électoraux comme produits de contextes politiques locaux}

\begin{tabular}{|lc|}
\hline & Coefficient $\boldsymbol{R}^{2}$ \\
\hline Extrême gauche & 0,82 \\
Abstention & 0,78 \\
Extrême droite & 0,77 \\
UMP & 0,76 \\
UDF & 0,76 \\
CPNT & 0,70 \\
Verts & 0,64 \\
PC & 0,60 \\
Socialistes & 0,55 \\
\hline
\end{tabular}

Exemple de lecture : on peut expliquer $82 \%$ des variations spatiales du vote d'extrême gauche à partir de la rếpartition géographique des donnểes INSEE utilisées pour la régression multiple. Ces variables INSEE sont prêsentẻes en fig. 3.

\section{J. Rivines CRESO - LMR ESO 6590 CNRS}

Figure 4 : Coefficient de détermination des régressions multiples entre les comportements électoraux et les indicateurs du recensement Regression coefficient of determination between electoral behaviours (1st turn of 2002's presidential election) and 1999's census data 


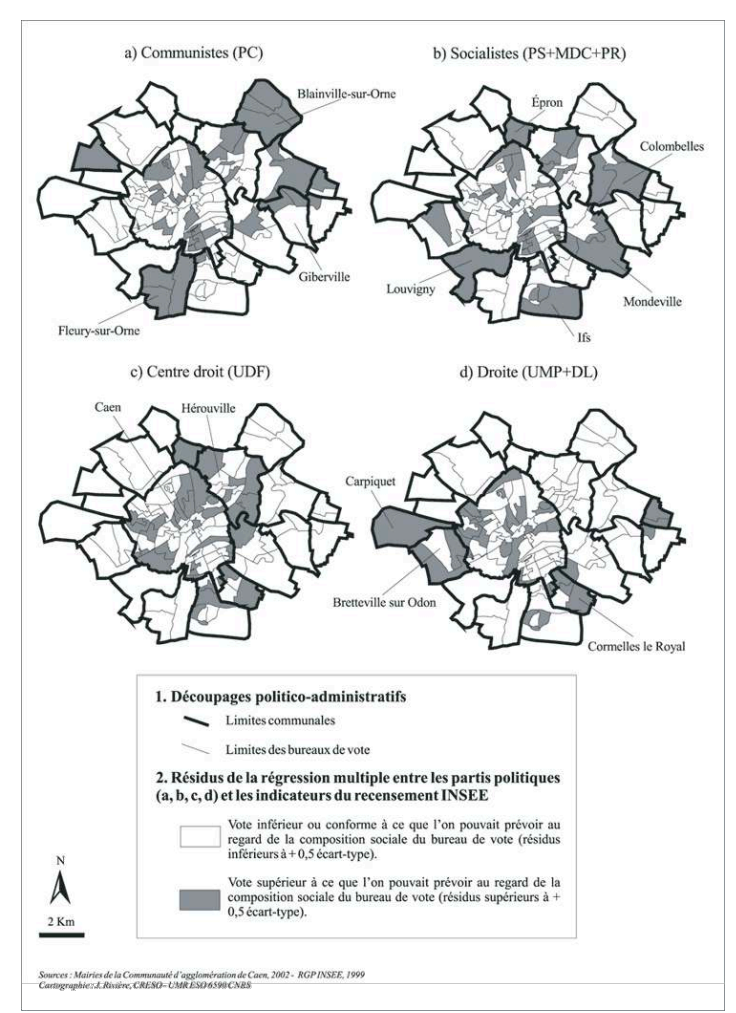

Figure 5 : Cartographie des résidus de régressions multiples entre les résultats des formations politiques gouvernementales (1er tour présidentielle 2002) et les indicateurs sociaux du recensement de 1999

Cartography of the residue's mathematic regression between governments party results (1st turn of 2002's presidential election) and 1999's census data

On a tenté de questionner, au sein de l'agglomération caennaise, le pouvoir explicatif des variables dites lourdes de la sociologie électorale. Au-delà des relations statistiques particulières établies entre tel vote et tel indicateur sociodémographique, le calcul d'une régression multiple entre chaque comportement et l'ensemble des variables INSEE mobilisées (ces variables et leurs modalités sont listées en fig. 3) devrait permettre à la fois de mesurer le pouvoir explicatif global de ces variables sur les votes et d'avancer dans la compréhension du processus de formation des identités politiques locales. L'analyse combinée des coefficients de détermination des régressions multiples (fig. 4) et la cartographie des résidus de ces régressions (fig. 5) fait ainsi apparaître des éléments essentiels.

Lorsque l'on classe les coefficients de détermination de ces différentes régressions multiples par ordre décroissant (fig. 4), il apparaît que selon le type d'attitude électorale, l'intensité de la relation entre la répartition spatiale des votes et la distribution géographique des variables sociales INSEE qui caractérisent les populations varie fortement. Ainsi, c'est avec les attitudes électorales dont on peut faire l'hypothèse qu'elles renvoient le plus à une forme de protestation politique que les coefficients $\mathrm{R}^{2}$ sont les plus élevés ( 0,82 pour l'extrême gauche, 0,77 pour l'extrême droite et 0,78 pour l'abstention), viennent ensuite de manière décroissante dans l'intensité les courants politiques de la droite (UMP, UDF, CPNT) puis les différentes familles de la gauche (Verts, PC, Socialistes). Cela signifie donc que la géographie des variables sociales dans l'agglomération caennaise permet largement de comprendre la répartition de certains comportements électoraux : l'abstention et les votes pour les deux familles extrêmes de 
l'échiquier politique français. C'est pour cette raison que les cartes des résidus de régression entre les indicateurs INSEE et ces comportements électoraux ne sont pas présentées. Par contre, le vote pour les candidats représentant les partis de gouvernement semble moins fortement lié à la présence de telle ou telle catégorie sociale, car les coefficients $\mathrm{R}^{2}$ sont moins élevés. Cependant, les configurations spatiales qui se dégagent des cartes de résidus des candidats à la présidentielle issus de ces partis de gouvernement (fig. 5) soulignent nettement les municipalités gérées par la formation politique dont émane chaque candidat ${ }^{4}$.

En ce qui concerne le PS par exemple, plusieurs mairies gérées par des socialistes (Colombelles, Mondeville, Ifs, Louvigny, etc.) apparaissent parmi les zones où les résidus sont positifs, c'est-à-dire là où le vote socialiste lors du scrutin présidentiel a été plus important que ce que le modèle de régression estimait d'après la structure sociale de la population résidente. Ceci est d'autant plus intéressant que l'on a vu auparavant que le vote pour les socialistes est celui le moins corrélé avec les indicateurs socio-économiques dont on a testé le caractère explicatif (on peut tout de même expliquer $55 \%$ des variations spatiales de ce vote dans le cadre de la régression multiple réalisée...). De la même manière, les zones de résidus positifs du candidat $\mathrm{R}$. Hue renvoient à des communes emblématiques de l'histoire industrielle de l'agglomération où le Parti Communiste $\mathrm{a}$, ou a eu, un rôle important dans la gestion municipale (Giberville, Fleury-sur-Orne, Blainville-sur-Orne). Une logique semblable est observable à droite puisque F. Bayrou recueille à Hérouville Saint-Clair (mairie remportée par l'UDF lors du scrutin municipal de 2001) plus de voix que la composition sociale de la commune ne le laissait penser. De même, les communes de Carpiquet, Cormelles-le-Royal et Bretteville-sur-Odon, qui ont toutes les trois un conseil municipal orienté à droite, font partie des espaces caractérisés par des résidus positifs en faveur des candidats de l'UMP.

Il semble donc bien que les élus locaux de l'agglomération participent à renforcer des identités politiques locales qui influencent les choix électoraux lors d'un scrutin de portée nationale comme l'élection présidentielle de 2002. L'essentiel reste néanmoins d'interroger les sociabilités qui produisent ces "effets de mairies", dynamiques qui renvoient plus largement au "neighbourhood effect", au "friends and neighbour effect » (Cox, 1969).

\section{Conclusion}

$\mathrm{Au}$ total, les différentes attitudes électorales face aux scrutins semblent intimement liées à des réalités sociales vécues par les habitants-électeurs de l'agglomération caennaise. Certes, le pouvoir explicatif des variables lourdes varie dans cette zone urbaine (selon les courants politiques, de $55 \%$ à $82 \%$ de la répartition géographique des votes est expliquée), mais on a pu montrer ensuite que des effets de contexte locaux concourent de manière notable aux choix des bulletins de vote lors de consultations électorales de portée nationale.

Le 29 mai dernier et alors que le scrutin renvoyait à l'échelle européenne, la répartition des suffrages au référendum épouse également les contours de la géographie sociale de l'agglomération. On observe ainsi de 60 à $70 \%$ de « oui » au sein des quartiers bourgeois du centre ouest de Caen et dans certaines communes périurbaines aisées de la couronne nord-ouest, contre 60 à $70 \%$ de «non » au cœur des quartiers paupérisés 
d'habitat social ou dans les communes de la «plaine rouge " dépeinte par A. Frémont (1986). En se basant sur les caractéristiques des départements français ${ }^{5}$ en terme de taux de chômage (et plus spécifiquement de chômage des femmes), de ménages monoparentaux ou d'allocataires du RMI, H. Le Bras (2005a, 2005b) construit d'ailleurs un système explicatif des résultats du dernier référendum européen autour de l'idée que «le non n'est ni de gauche ni de droite, mais précaire ». Ces éléments tendent à infirmer certaines conclusions antérieures du même démographe (2002) qui avançait que la géographie des votes s'était autonomisée par rapport aux conditions "matérielles et professionnelles", conditions qui déterminent pourtant l'existence matérielle quotidienne de chaque citoyen.

On peut néanmoins suivre H. Le Bras lorsqu'il interroge l'impact de "l'anthropologie du voisinage ", et de la dimension "interactive et communicationnelle» dans le processus (localisé) de formation des opinions politiques. En 1996, la revue Political Geography a d'ailleurs ouvert ses colonnes à un débat pluridisciplinaire autour d'un texte de J. Agnew (1996) intitulé "Mapping politics: how context counts in electoral geography ». L'auteur entend y dépasser la mise en évidence des effets de voisinage pour proposer un apport plus global de la géographie dans le champ des disciplines qui traitent des questions électorales et politiques. Cette dernière proposition est particulièrement intéressante car, bien que la géographie anglo-saxonne soit largement influencée par les courants de l'analyse spatiale, les propos de J. Agnew encouragent les chercheurs à explorer des contextes locaux en montrant qu'il s'agit bien de productions des sociétés. Dans sa contribution au débat sur la place des contextes dans la compréhension du vote, C. Flint (1996) rejoint d'ailleurs J. Agnew et ouvre des perspectives en postulant que le manque de liaisons entre la conceptualisation de la notion de contexte et l'approche micro-géographique ne constitue pas une crise pour la géographie électorale, mais bien une opportunité.

\section{BIBLIOGRAPHIE}

AGNEW (J. E.), 1996. - « Mapping politics: how context counts in electoral geography », Political Geography, vol. 15, n², p. 129-145.

BADARIOTTI (D.), 1994. - Ville et vote. Urbanisme et géographie électorale à Strasbourg sous la Cinquième République, Thèse de doctorat en géographie, Université de Strasbourg, 266 p.

Bon (F.), CheYlan (J.-P.), 1988. - La France qui vote, Paris, Hachette, 464 p.

BRÉCHON (P.), CAUTRÈS (B.), 1987. - « L'inscription sur les listes électorales : indicateur de socialisation ou de politisation? ", Revue française de science politique, $\mathrm{n}^{\circ} 4$, p. 502-525.

Bussi (M.), 1992. - « Géographie politique des villes normandes », Études Normandes, n 4, p. 4-20.

Bussi (M.), ColANGe (C.), Gosset (J.-P.), 2005. - « Réalignements partisans face à la construction européenne », Cybergéo, 3 juin 2005, 14 p. 
Cox (K. R.), 1969. - « The voting decision in a spatial context », Progress in Geography, n 1, p. 81-117.

CAUTRÈS (B.), MAYER (N.), 2004. - Le nouveau désordre électoral. Les leçons du 21 avril 2002, Paris, Presses de Sciences Po, $412 \mathrm{p}$.

FLINT (C.), 1996. - « Wither the individual, wither the context?», Political geography, vol. 15, n² p. $45-50$.

FRÉMONT (A.), 1986. - « La Basse-Normandie », dans LacosTE (Y.) (dir.), Géopolitique des régions françaises- Tome 1 : La façade occidentale, Paris, Fayard, p. 26-164.

GIRAULT (F.), 2000. - Le vote comme expression territoriale des citadins - Contribution à l'étude des ségrégations urbaines, Université de Rouen, thèse de Doctorat en Géographie, 2 vol., 504 p. Goux (D.), MAURIN (E.), 2004. - « Anatomie sociale d'un vote », Le Monde, 14 avril, p. 9.

LAURENT (A.), 1997. - « Vote et ancienneté des attaches résidentielles », dansDUPOIRIER (E.), PARODI (J.-C.), Les indicateurs socio-politiques aujourd'hui, Paris, L'Harmattan, p. 335-350.

LE BRAS (H.), 2002. - Une autre France, Paris, Odile Jacob, 223 p.

-, 2005a. - «Le non n'est ni de gauche ni de droite, il est précaire », Libération, Cahier spécial référendum, $1^{\text {er }}$ juin 2005, p. E4 du supplément.

-, 2005b. - « La mémoire des territoires », dans FINCHELSTEIN (G.) (éd.), Le jour où la France a dit non. Comprendre le référendum du 29 mai 2005, Paris, Plon/Fondation Jean-Jaurès, 153 p, p. 56-77.

LÉVY (J.), 1994. - L'espace légitime. Sur la dimension spatiale de la fonction politique, Paris, Presses de la FNSP, $442 \mathrm{p}$.

MAHÉ (A.), 1968. - Géographie électorale de l'agglomération caennaise, mémoire de Maîtrise sous la direction de P. Brunet, Université de Caen, 178 p.

RAPETTI (D.), 1985. - Vote et société dans la région nantaise. Étude de géographie électorale 1945-1983, Paris, CNRS, $210 \mathrm{p}$.

RHEIN (C.), 1994. - « La ségrégation et ses mesures », BRUN (J.), RHEIN (C.) (dir.), La ségrégation dans la ville, Paris, L'Harmattan, coll. « Villes et territoires », 289 p., p. 121-161.

SANGUIN (A.-L.), 1977. - La géographie politique, Paris, PUF, coll. « Le Géographe », n² 24, 183 p.

SIEGFRIED (A.), 1913. - Tableau politique de la France de l'Ouest, Paris, A. Colin, 536 p.

TROUfleAu (P.), 1995. - « Géographie des comportements électoraux en Eure et Loire : essai d'explication socio-économique et problèmes méthodologiques ", L'Espace géographique, $\mathrm{n}^{\circ} 2, \mathrm{p}$. 155-168.

SERONDE-BABONAUX (A.-M.), 1983. - « Les comportements électoraux dans l'agglomération de Caen : 1974/1981 », dans Noin (D.) (éd.), Actes du colloque de Géographie sociale de Lyon, Paris, p. 374-382.

\section{NOTES}

1. Dans la mesure où l'on fait l'hypothèse - généralement admise dans les disciplines qui traitent des questions politiques - que c'est lors du scrutin présidentiel que la position sociale joue le plus dans le comportement d'un électeur, ce sont les résultats de cette consultation électorale qui 
seront principalement mobilisés dans cet article. Afin de mettre en évidence des tendances générales, les candidats de l'élection de 2002 sont regroupés en familles politiques.

2. Ouest-France, 23 avril 2002.

3. Ce qui n'est pas surprenant tant la présence des « étudiants » est très fortement corrélée avec celle des «18-24 ans» dans l'agglomération caennaise. Par ailleurs, la question de la noninscription chez les étudiants a fait l'objet d'enquêtes spécifiques (Bréchon et Cautrès, 1987) dans la ville universitaire de Grenoble où les étudiants représentent un fort pourcentage de la population communale.

4. D'ailleurs, l'extrême gauche ou l'extrême droite ne gèrent pas de municipalité dans l'agglomération caennaise.

5. Cependant il est clair qu'une telle analyse de corrélations à l'échelle départementale présente un risque évident d'erreur écologique (Bussi et al., 2005).

\section{RÉSUMÉS}

À la différence des sondages nationaux basés sur des enquêtes individuelles, l'analyse des votes fait apparaître des différences spatialisées à des échelles géographiques très fines, notamment en milieu urbain. Dans cette perspective, cet article tente d'explorer les relations qui lient les comportements électoraux et les caractéristiques sociales des habitants de l'agglomération caennaise lors de l'élection présidentielle de 2002. À l'aide de traitements quantitatifs et d'une approche cartographique, on se propose de montrer que l'analyse des choix électoraux lors d'un scrutin national implique de prendre en compte à la fois la structure sociale urbaine et le poids du contexte politique local.

Unlike the national public opinion poll. built on individual surveys, the analysis based on electoral results shows the existence of spatial differences on large geographical scales, more particularly in urban areas. In this perspective, this paper tries to explore the relationships between electoral behaviours and social characteristics of inhabitants of the Caen urban area on the occasion of the 2002's presidential election. Using quantitative methods and cartographic approach, we intend to show that the analysis of electoral choices in a national election imply to take account the social urban structure and the local political context.

\section{INDEX}

Mots-clés : approche contextualisée, agglomération, division sociale

Index géographique : Basse-Normandie, Caen, France

Keywords : contextual analysis, agglomeration, social divisions 
AUTEUR

JEAN RIVIÈRE

CRESO - UMR 6590 CNRS ESO, Université de Caen Basse-Normandie, MRSH, Esplanade de la Paix, BP 5186, 14032 Caen cedex, France,

jean.riviere@unicaen.fr 